\title{
Validación del instrumento autoevaluación de habilidades del aprendizaje: un pronóstico del rendimiento escolar en estudiantes universitarios
}

\section{Validation of the learning skills self-assessment instrument: a forecast of school performance in universitary students}

\author{
RÍOS-VALLES, José Alejandro †ै*, LARES-BAYONA, Edgar Felipé, MARTÍNEZ-MARTÍNEZ, \\ Patricia Lorena" y FERNÁNDEZ-ESCÁRZAGA, Jaime
}

\author{
Instituto de Investigación Científica de la Universidad Juárez del Estado de Durango. Universidad y Fanny Anitua SN, Zona \\ Centro, 34000 Durango, Dgo. \\ "Facultad de Psicología y Terapia de la Comunicación Humana de la Universidad Juárez del Estado de Durango. Del \\ Guadiana 501, Fraccionamiento Ciudad Universitaria, 34120 Durango, Dgo.
}

ID $1^{\mathrm{er}}$ Autor: José Alejandro, Ríos-Valles / ORC ID: 0000-0002-8407-3017, Researcher ID Thomson: X-3209-2018, CVU CONACYT ID: 313266

ID $1^{\text {er }}$ Coautor: Edgar Felipe, Lares-Bayona

ID $2{ }^{\text {do }}$ Coautor: Patricia Lorena, Martínez-Martínez / ORC ID: 0000-0003-4860-1625, CVU CONACYT ID: 221255

ID $3^{\text {er }}$ Coautor: Jaime, Fernández-Escárzaga

DOI: $10.35429 / J U P .2019 .10 .3 .28 .36$

Recibido: 15 de Octubre, 2019; Aceptado 23 de Diciembre, 2019

\section{Resumen}

Las habilidades del aprendizaje se reflejan en el desempeño escolar del estudiante, el cual es expresado por la calificación asignada por el profesor o el promedio obtenido por el estudiante. Los problemas del aprendizaje suelen manifestarse por el bajo promedio escolar. Objetivos: Validar y correlacionar un instrumento para la autoevaluación de las habilidades para el aprendizaje y el promedio escolar en estudiantes de la Universidad Juárez del Estado de Durango. Metodología: Investigación exploratoria, transversal, descriptiva, correlacional y de validación, con muestreo no probabilístico, por conveniencia previa firma del consentimiento informado. Contribución: el instrumento de autoevaluación de las habilidades para el aprendizaje es fiable y permite identificar el nivel de eficiencia con el que se autoevalúan los participantes en la muestra estudiada, por lo que puede ser utilizado para orientar las necesidades de atención educativa en estudiantes de educación superior.

Habilidades del aprendizaje, Autoevaluación, Estudiantes universitarios

\begin{abstract}
Learning skills are reflected in the student's school performance, which is expressed by the teacher's assigned grade or the average earned by the student. Learning problems often manifest themselves by low school averages. Objectives: Validate and correlate an instrument for the self-assessment of learning skills and school average in students of the Juarez University of Durango State. Methodology: Exploratory, cross-cutting, descriptive, correlational and validation research, with non-probabilistic sampling, for convenience upon signature of informed consent. Contribution: The selfassessment tool for learning skills is reliable and allows to identify the level of efficiency with which participants in the studied sample are self-assessed, so it can be used to guide the needs educational care in higher education students.
\end{abstract}

Learning Skills, Self-Assessment, Universitary students

Citación: RÍOS-VALLES, José Alejandro, LARES-BAYONA, Edgar Felipe, MARTÍNEZ-MARTÍNEZ, Patricia Lorena y FERNÁNDEZ-ESCÁRZAGA, Jaime. Validación del instrumento autoevaluación de habilidades del aprendizaje: un pronóstico del rendimiento escolar en estudiantes universitarios. Revista de Políticas Universitarias. 2019. 3-10: 28-36.

\footnotetext{
* Correspondencia al Autor (Correo electrónico: alexriva@ hotmail.com)

$\dagger$ Investigador contribuyendo como primer autor.
} 


\section{Introducción}

Las habilidades del aprendizaje se deben considerar como responsables del desempeño escolar, el cual se ha definido como el "cumplimiento de las metas, logros u objetivos establecidos en el programa o asignatura (VelezVan, 2005), o como el nivel de logro que puede alcanzar un estudiante en una $\mathrm{o}$ varias asignaturas (Barceló, 2006), logro que vincula más con la capacidad que con el desempeño del propio estudiante (Navarro, 2003).

El desempeño escolar es una variable compleja que depende de circunstancias internas y externas, lo que permite que pueda ser predicho por características internas como las cognoscitivas (inteligencia) y del comportamiento (personalidad, motivación). (Cupani, 2013), es decir, el desempeño académico es complejo y, además multifactorial porque supone no sólo desarrollo cognoscitivo sino también aspectos sociales, económcicos y culturales, por dar un ejemplo de características externas (Navarro, 2003).

Como evidencia del aprendizaje, el desempeño escolar puede ser expresado por la calificación asignada por el profesor o el promedio obtenido por el estudiante. El promedio resume el rendimiento escolar y los problemas escolares relacionados con el bajo rendimiento y la deserción académica son de interés para padres, maestros y profesionales en el campo educativo y pueden estar relacionados con aspectos como la salud física y mental (Palacios, 2007).

El aprendizaje depende de la adecuada función del sistema nervioso y el proceso de aprender involucra la organización de redes neurales por lo que se debe considerar que los trastornos del aprendizaje se manifiestan la mayoría de las veces por bajo promedio escolar.

Echavarri demuestra la existencia de relaciones positivas, moderadas y grandes, entre habilidades cognitivas y rendimiento académico tanto para hombres como para mujeres, encontrando además que estas relaciones fueron mayores en las mujeres que en los hombres (Echavarri M. G., 2007).
Barceló identifica que el rendimiento académico no está directamente relacionado con déficits a nivel de las habilidades ejecutivas, pero considera que sí podría estarlo a nivel del lenguaje $\mathrm{y}$ de los antecedentes familiares, psicológicos y académicos en estudiantes universitarios (Barceló, 2006).

La medición de la inteligencia y las habilidades cognitivas han sido estudiadas por diferentes autores desde principios del siglo pasado. La evaluación de este constructo consiste en muestrear una amplia variedad de habilidades y combinar los puntajes obtenidos en un solo puntaje compuesto por la suma de los puntos obtenidos en cada una de las habilidades muestreadas, que generalmente es referido como coeficiente de inteligencia. (Echavarri M. G., 2007). En todo estudiante universitario la integridad fisiológica de las funciones mentales relacionadas con el aprendizaje es indispensable para un promedio escolar satisfactorio para promover la eficiencia terminal óptima. Esta última es un importante indicador que determina la eficiencia de los procesos educativos en las universidades y que la Secretaría de Educación Pública la define como el número de estudiantes que terminan un nivel educativo de manera regular (dentro del tiempo ideal establecido) y el porcentaje de estudiantes que lo culminan extemporáneamente (Secretaria de Educacion Publica, 2014).

La eficiencia terminal en educación superior tiene un impacto económico importante para el país y para las instituciones educativas pues en el sexto informe presidencial del Gobierno de México se indica que el ciclo escolar del 2017-2018 la eficiencia terminal a nivel de licenciatura se encuentra en el $69.4 \%$ y el costo anual por alumno es de 79.9 miles de pesos (Gobierno de los Estados Unidos Mexicanos. Presidencia de la República., 2018). Considerando a todas las unidades académicas de la Universidad Juárez del Estado de Durango, la eficiencia terminal durante el ciclo escolar del 2016 se registró una eficiencia terminal del $47 \%$ en el primer semestre y del $44 \%$ en el segundo semestre, en el 2017 respectivamente la eficiencia terminal fue del $47 \%$ y $43 \%$ y en el 2018 los valores fueron del 55\% y 50\%. (Universidad Juárez del Estado de Durango, 2017) (Universidad Juárez del Estado de Durango, 2018) (Universidad Juárez del Estado de Durango, 2019). 
Particularmente en los estudiantes de la Licenciatura en Terapia de la Comunicación Humana, la eficiencia terminal en el primer y segundo semestre de los últimos tres años respectivamente muestra; en el 2016 el $74 \%$ y $37 \%$, en el 2017 el $5 \%$ y el $3 \%$ y en el 2018 el $54 \%$ y $34 \%$ (Universidad Juárez del Estado de Durango, 2017) (Universidad Juárez del Estado de Durango, 2018) (Universidad Juárez del Estado de Durango, 2019)

Por todo lo anteriormente descrito y particularmente observando que durante el primer y segundo semestre del ciclo escolar del 2018, la eficiencia terminal del total de los alumnos de la Universidad Juárez del Estado de Durango fue del 55 y $50 \%$ y en alumnos de la Licenciatura en Terapia de la Comunicación Humana del $54 \%$ y $34 \%$ respectivamente. En ambos casos la eficiencia terminal es menor de la media nacional que es del $69.4 \%$ a nivel licenciatura (Gobierno de los Estados Unidos Mexicanos. Presidencia de la República., 2018) lo que implica un gran gasto económico, por lo que resulta importante buscar estrategias que ayuden a identificar instrumentos confiables que orienten la necesidades de atención educativa que permitan una mejor eficiencia terminal. Por ello se pretende saber si la autoevaluación de las habilidades del aprendizaje constituye un instrumento confiable para identificar la eficiencia de estas habilidades en los estudiantes universitarios y que pudiese ser empleado para identificar que habilidades del aprendizaje requieren ser habilitadas u optimizadas en los estudiantes para obtener un mejor promedio escolar y que esto se refleje positivamente en la eficiencia terminal.

\section{Planteamiento del problema}

La baja eficiencia terminal en estudiantes de la Universidad Juárez del Estado de Durango es un problema que debe preocupar a la comunidad universitaria, pues el rezago escolar evidenciado por la baja eficiencia terminal representa un costo económico y social importante para cualquier país. De acuerdo a la estimación del VI Informe de Gobierno de la Presidencia de los Estados Unidos Mexicanos (2018), cada alumno en educación superior representa un gasto de 79.9 mil pesos anuales, si se considera, de acuerdo a la eficiencia terminal del 55 y $50 \%$ referida en el anuario estadístico del 2018 de la UJED y que la población total de nivel licenciatura (Escolarizada y virtual) en ese ciclo escolar fue de 13,823 estudiantes.
Considerando el $50 \%$ de la matrícula de licenciatura y virtual de la UJED en 2018, equivale a 6911 alumnos, lo cual de acuerdo a la estimación del costo anual por estudiante en Licenciatura, representa un costo de 552 millones 228 mil pesos para el país en la Universidad Juárez del Estado de Durango por efecto de la eficiencia terminal referida.

En la actualidad, los programas de planeación educativa no han surtido un efecto de impacto inmediato para resolver la problemática de eficiencia terminal. Sin embargo, instrumentos considerados para diagnosticar los problemas educativos de tipo cognitivo y estructural, no han sido del todo validados para la población de la UJED y población similar en los niveles de estudio de licenciatura.

No contar con instrumentos de medición confiables y validados para el diagnóstico de habilidades de aprendizaje en una población de estudiantes universitarios, es un problema educativo para los departamentos de planeación, dado que no tienen las herramientas de evaluación cognitiva confiables que inciden en la eficiencia terminal como un pronóstico para el aseguramiento del rendimiento escolar.

\section{Objetivo general}

Validar el instrumento Autoevaluación de Habilidades del Aprendizaje y correlacionar con el rendimiento escolar en estudiantes de la Universidad Juárez del Estado de Durango, México.

\section{Objetivos específicos}

1. Validar mediante el Análisis Factorial Exploratorio, al instrumento de Autoevaluación de Habilidades de Aprendizaje en estudiantes universitarios de la Facultad de Psicología y Terapia de la Comunicación Humana

2. Identificar la habilidad para el aprendizaje con mayor correlación con el promedio escolar.

3. Establecer la habilidad del aprendizaje con menor correlación con el promedio escolar.

4. Determinar que habilidad del aprendizaje no correlaciona con el promedio escolar.

RÍOS-VALLES, José Alejandro, LARES-BAYONA, Edgar Felipe, MARTÍNEZ-MARTÍNEZ, Patricia Lorena y FERNÁNDEZESCÁRZAGA, Jaime. Validación del instrumento autoevaluación de habilidades del aprendizaje: un pronóstico del rendimiento escolar en estudiantes universitarios. Revista de Políticas Universitarias. 2019 
5. Conocer que habilidad del aprendizaje tiene mayor correlación con las demás variables de habilidades del aprendizaje.

\section{Marco teórico}

La validez del constructo se considera fundamental en las teorías psicométricas dentro del campo de la psicología y las ciencias de la conducta.

Los trabajos de Cyril-Hoyt (1941) y Lous-Guttman (1945) desarrollaron las bases de validación de ítems en escala ordinal dentro de la teoría psicométrica.

Josep Lee Cronbach (1951) define lo que se considera aún los procesos de medición para determinar la confiabilidad de constructos de carácter psicosocial con ítems de escala tipo Likert, mediante medidas de correlación marginal comparativa inter-ítem, y así explicar el grado de agrupación de los ítems con variables latentes que explican en su conjunto términos o cuestiones como depresión, ideación suicida, valores, desarrollo saludable, sexualidad, ansiedad, etc. (Cronbach L. J., 1951) (Cronbach L. J., 1972).

Autores como Nunnally definen la confiabilidad como el grado en que las mediciones se pueden repetir y que cualquier influencia aleatoria tiende a generar diferentes mediciones en distintas ocasiones, por lo tanto, Nunally considera que el objetivo es la ardua búsqueda de la medida del error (Nunnally, 1967).

El estadístico de confiabilidad definido por Joseep Lee Cronbach (1951), es sensible a los errores producidos por la población de estudio, por lo que trata de amortiguar dichos errores implementando una escala tipo Likert, a diferencia de Guttman (1945) y Hoyt (1941) en sus escalas de tipo ordinal.

De esta manera la Validez es una relación plausible entre lo que el instrumento mide y lo que realmente se quiere medir. El Análisis Factorial Exploratorio (AFE) representa las técnicas más frecuentes para la validación de constructos empleando análisis multivariante que permite identificar las estructuras subyacentes de los ítems, con procesos de cálculo en la identificación de variables latentes determinadas en el análisis (Cronbach, 1951).

\section{Material y métodos}

Se realizó una investigación exploratoria, transversal, descriptiva, correlacional y de validación, de un instrumento para la autoevaluación de habilidades para el aprendizaje y auto-referencia del promedio escolar en una muestra de 123 estudiantes de la UJED de la Licenciatura en Terapia de la Comunicación Humana seleccionados en un muestreo no probabilístico, por accidente y por conveniencia.

Criterios de inclusión:

Alumnos de la Licenciatura en Terapia de la Comunicación Humana de la UJED que estuviesen presentes en su unidad académica el día de aplicación del instrumento de autoevaluación de habilidades para el aprendizaje y que aceptaron participar voluntariamente previa firma de consentimiento informado.

\section{Criterios de exclusión:}

Alumnos de la UJED que derivado de circunstancias de índole personal no estuviesen dispuestos a que se les aplique el instrumento de autoevaluación de habilidades para el aprendizaje.

\section{Criterios de eliminación:}

Alumnos que proporcionen información incompleta.

\section{Aspectos éticos}

De acuerdo al artículo 17 de la la Ley General de Salud en México la investigación realizada no tiene riesgo alguno para la salud y en consideración al artículo 20 de la misma Ley, se respeta el consentimiento informado de todos los participantes y los establecido en la Declaración de Helsinki.

\section{Procedimiento}

Se acudió a visitar alumnos de la Licenciatura en Terapia de la Comunicación Humana de la Universidad Juárez del Estado de Durango (UJED) a quienes se puso a su consideración la invitación a participar en forma voluntaria y anónima a llenar la encuesta de autoevaluación de habilidades para el aprendizaje a lo cual accedieron 123 alumnos.

RÍOS-VALLES, José Alejandro, LARES-BAYONA, Edgar Felipe, MARTÍNEZ-MARTÍNEZ, Patricia Lorena y FERNÁNDEZESCÁRZAGA, Jaime. Validación del instrumento autoevaluación de habilidades del aprendizaje: un pronóstico del rendimiento escolar en estudiantes universitarios. Revista de Políticas Universitarias. 2019 
La información recabada se organizó en una base de datos y para su análisis estadístico se empleó el software SPSS versión 20 (Statistical Package for the Social Sciences).

\section{Resultados}

\section{Características sociodemográficas}

El número de participantes fue de 123 alumnos, predominando las personas del sexo femenino con $74.8 \%$ de la muestra (ver Tabla 1 ).

\begin{tabular}{|l|r|r|}
\multicolumn{1}{|c|}{ Sexo } & \multicolumn{1}{c|}{ Frecuencia } & \multicolumn{1}{c|}{ Porcentaje } \\
\hline Masculino & 31 & 25.2 \\
\hline Femenino & 92 & 74.8 \\
\hline Total & 123 & 100.0 \\
\hline
\end{tabular}

Tabla 1 Sexo

La edad de los participantes fluctuó entre los 17 y los 24 años predominando la edad de 20 años con $28.5 \%$ de la población (ver tabla 2 )

\begin{tabular}{|r|r|r|r|}
\hline \multicolumn{2}{|c|}{ Edad Frecuencia Porcentaje } & \multicolumn{1}{c|}{$\begin{array}{l}\text { Porcentaje } \\
\text { acumulado }\end{array}$} \\
\hline 17 & 2 & 1.6 & 1.6 \\
\hline 18 & 25 & 20.3 & 22.0 \\
\hline 19 & 25 & 20.3 & 42.3 \\
\hline 20 & 35 & 28.5 & 70.7 \\
\hline 21 & 16 & 13.0 & 83.7 \\
\hline 22 & 12 & 9.8 & 93.5 \\
\hline 23 & 7 & 5.7 & 99.2 \\
\hline 24 & 1 & .8 & 100.0 \\
\hline Total & 123 & 100.0 & \\
\hline
\end{tabular}

Tabla 2 Edad

La distribución del estado civil de los participantes muestra predominio de solteros con un $82.9 \%$ de la muestra evaluada, como se observa en la tabla 3.

\begin{tabular}{|l|r|r|r|}
\hline \multicolumn{2}{|l|}{ Edo. Civil Frecuencia Porcentaje } & \multicolumn{2}{c|}{$\begin{array}{l}\text { Porcentaje } \\
\text { acumulado }\end{array}$} \\
\hline Soltero & 102 & 82.9 & 82.9 \\
\hline Casado & 8 & 6.5 & 89.4 \\
\hline Unión & 9 & 7.3 & 96.7 \\
Libre & & & 99.2 \\
\hline Divorciado & 3 & 2.4 & 100.0 \\
\hline Separado & 1 & .8 & \\
\hline Total & 123 & 100.0 & \\
\hline
\end{tabular}

Tabla 3 Estado civil

Los semestres que cursaban los alumnos encuestados fueron de primero a octavo, con el mayor porcentaje en los alumnos del cuarto semestre con un $41.5 \%$ (ver tabla 4 ).

\begin{tabular}{|r|r|r|r|}
\hline \multicolumn{2}{|c|}{ Semestre Frecuencia } & Porcentaje & \multicolumn{1}{c|}{ Porcentaje } \\
\hline 1 & 37 & 30.1 & 30.1 \\
\hline 2 & 8 & 6.5 & 36.6 \\
\hline 3 & 11 & 8.9 & 45.5 \\
\hline 4 & 51 & 41.5 & 87.0 \\
\hline 5 & 4 & 3.3 & 90.2 \\
\hline 6 & 3 & 2.4 & 92.7 \\
\hline 7 & 3 & 2.4 & 95.1 \\
\hline 8 & 6 & 4.9 & 100.0 \\
\hline Total & 123 & 100.0 & \\
\hline
\end{tabular}

Tabla 4 Semestre

En cuanto al estatus escolar de los alumnos participantes predominó el Regular, es decir alumnos que no han recursado materias con un $81.3 \%$ (ver tala 5 ).

\begin{tabular}{|c|c|c|c|}
\hline & \multicolumn{2}{|c|}{ Frecuencia Porcentaje } & Porcentaje \\
\hline Válidos $\begin{array}{l}\text { No } \\
\text { regular }\end{array}$ & 23 & 18.7 & 18.7 \\
\hline Regular & 100 & 81.3 & 100.0 \\
\hline Total & 123 & 100.0 & \\
\hline
\end{tabular}

Tabla 5 Estatus escolar

El promedio escolar de la muestra estudiada mostró una media de 8.53 y desviación estándar de 0.6. Con los valores anteriores se dividió la muestra en dos grupos, los que presentaron promedio menor a la media grupal y los que presentaron promedio igual o mayor a la media. (ver Tablas 6 y 7 )

\begin{tabular}{|l|r|r|r|r|r|}
\hline & \multicolumn{3}{|c|}{ Mínimo Máximo } & Media & $\begin{array}{c}\text { Desv. } \\
\text { típ. }\end{array}$ \\
\hline $\begin{array}{l}\text { Promedio } \\
\text { escolar }\end{array}$ & 123 & 6.90 & 9.80 & 8.53 & .60 \\
\hline
\end{tabular}

Tabla 6 Promedio escolar

\begin{tabular}{|c|c|c|}
\hline & Frecuencia & Porcentaje \\
\hline$<=8.52$ & 58 & 47.2 \\
\hline $8.53+$ & 65 & 52.8 \\
\hline Total & 123 & 100.0 \\
\hline
\end{tabular}

Tabla 7 PROMESC (agrupado)

La media y desviación estándar del Promedio escolar autorreferido (PROMESC) y la autoevaluación de las Habilidades para el Aprendizaje se muestran en la tabla 8. 


\begin{tabular}{|l|r|r|r|}
\hline & \multicolumn{1}{|c|}{ Media } & Desviación estándar & N del análisis \\
\hline PROMESC & 8.54 & .60 & 123 \\
\hline ATENCION & 7.37 & 1.47 & 123 \\
\hline ENTESC & 7.80 & 1.27 & 123 \\
\hline ENTLEO & 7.75 & 1.38 & 123 \\
\hline MEMAUD & 7.59 & 1.62 & 123 \\
\hline MEMVIS & 8.20 & 1.24 & 123 \\
\hline CALCMAT & 6.79 & 1.93 & 123 \\
\hline ESCRIBIR & 7.91 & 1.60 & 123 \\
\hline HABLAR & 8.13 & 1.30 & 123 \\
\hline LEER & 8.37 & 1.41 & 123 \\
\hline PENSAR & 8.52 & 1.17 & 123 \\
\hline
\end{tabular}

Tabla 8 PROMESC y Habilidades para el Aprendizaje

La confiabilidad del instrumento de autoevaluación de habilidades para el aprendizaje (HA) aplicado a la muestra de alumnos participantes y la autorreferencia del promedio escolar mostró un Alfa de Cronbach de 0.847 (ver tabla 9).

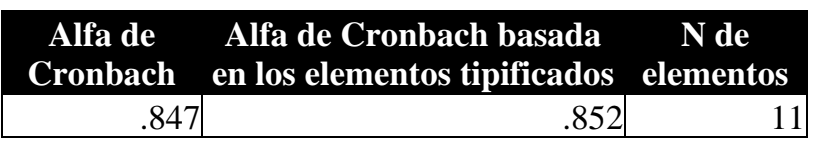

Tabla 9 Confiabilidad de autoevaluación de HA y PE

El análisis de adecuación muestral de Kaiser-Meyer-Olkin mostró un valor optimo para el análisis factorial de las variables por tener un valor de 0.858 y la prueba de esfericidad de Bartlett fue significativa por ser menor al alfa de 0.05 lo que demuestra que es factible realizar un análisis factorial a la distribución de los datos (ver tabla 10).

$\begin{aligned} & \text { Medida de adecuación muestral de Kaiser- } \\
& \text { Meyer-Olkin. }\end{aligned}$
\begin{tabular}{|l|l|r|}
\hline $\begin{array}{l}\text { Prueba de esfericidad de } \\
\text { Bartlett }\end{array}$ & $\begin{array}{l}\text { Chi-cuadrado } \\
\text { aproximado }\end{array}$ & 433.228 \\
\cline { 2 - 3 } & gl & 55 \\
\cline { 2 - 3 } & Sig. & .000 \\
\hline
\end{tabular}

Tabla 10 KMO y prueba de Bartlett

Dado el nivel de fiabilidad global del Alfa de Cronbach de 0.852, en el análisis del alfa individual por cada variable no se observa necesario quitar algún elemento para incrementar el Alfa, por lo que se decide mantener los11 elementos (ver Tabla 11).

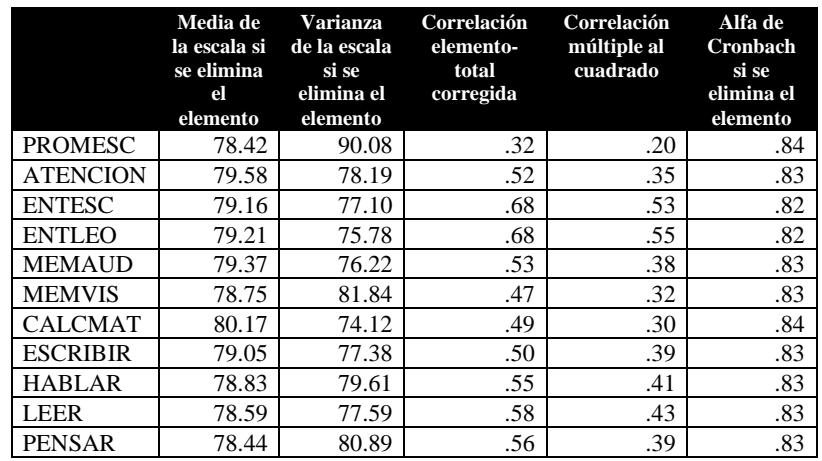

Tabla 11 Estadísticos total-elemento

\section{Análisis de componentes principales}

La matriz de componentes principales sin rotación muestra dos variables latentes. La primera de ellas, conformada por los 10 items del instrumento de autoevaluación de las habilidades para el aprendizaje y el promedio escolar, estableciéndose un modelo predictivo de la autoevaluación de las habilidades para el aprendizaje, al cual se le ha denominado APRENDIZAJE EFICIENTE y que es explicado por la eficiencia con la que se entiende lo que se escucha y lo que se lee, seguido de la eficiencia en la lectura, el pensar, la memoria auditiva, el hablar, la atención, el escribir, la memoria visual, el cálculo matemático y el promedio escolar; y la segunda variable latente es conformada por el mismo número de ítems que explican la primer variable latente por lo que se le identificó como PROMEDIO ESCOLAR MEJORADO (ver Tabla 12).

\begin{tabular}{|l|r|r|}
\hline \multicolumn{3}{c}{ Componente } \\
\hline ENTESC & .781 & .153 \\
\hline ENTLEO & .778 & .137 \\
\hline LEER & .682 & -.339 \\
\hline PENSAR & .662 & -.176 \\
\hline MEMAUD & .645 & -.131 \\
\hline HABLAR & .639 & -.398 \\
\hline ATENCION & .625 & .319 \\
\hline ESCRIBIR & .592 & -.449 \\
\hline MEMVIS & .585 & .348 \\
\hline CALCMAT & .581 & .125 \\
\hline PROMESC & .411 & .610 \\
\hline
\end{tabular}

Tabla 12 Matriz de componentes ${ }^{\mathrm{a}}$

Lo mostrado en la tabla 12 se corrobora en el gráfico de sedimentación. 


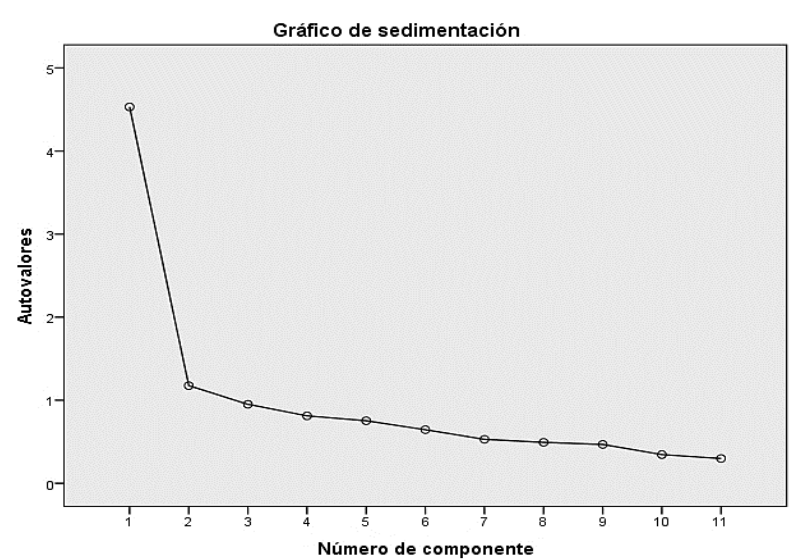

Gráfico 1

En la grafica de sedimentación anterior los dos primeros elementos del análisis de componentes principales, que son las variables latentes Aprendizaje Eficiente y Promedio Escolar Mejorado, explican el 51.879\% de la varianza del instrumento de autoevaluación de habilidades para el aprendizaje, como se puede ver en la tabla 13.

\begin{tabular}{|c|c|c|c|c|c|}
\hline \multirow[t]{2}{*}{ Componente } & \multicolumn{3}{|c|}{ Autovalores iniciales } & \multicolumn{2}{|c|}{$\begin{array}{c}\text { Sumas de las } \\
\text { saturaciones al } \\
\text { cuadrado de la } \\
\text { extracción }\end{array}$} \\
\hline & Total & $\begin{array}{c}\% \text { de la } \\
\text { varianza }\end{array}$ & $\begin{array}{c}\% \\
\text { acumulado }\end{array}$ & Total & $\begin{array}{c}\% \text { de la } \\
\text { varianza }\end{array}$ \\
\hline 1 & 4.531 & 41.192 & 41.192 & 4.531 & 41.192 \\
\hline 2 & 1.176 & 10.687 & 51.879 & 1.176 & 10.687 \\
\hline 3 & .951 & 8.646 & 60.525 & & \\
\hline 4 & .812 & 7.381 & 67.907 & & \\
\hline 5 & .753 & 6.846 & 74.752 & & \\
\hline 6 & .645 & 5.861 & 80.613 & & \\
\hline 7 & .529 & 4.810 & 85.423 & & \\
\hline 8 & .493 & 4.478 & 89.901 & & \\
\hline 9 & .467 & 4.250 & 94.151 & & \\
\hline 10 & .345 & 3.138 & 97.288 & & \\
\hline 11 & .298 & 2.712 & 100.000 & & \\
\hline
\end{tabular}

Tabla 13 Varianza total explicada

\section{Correlaciones}

Las Correlaciones observadas entre las variables de autoevaluación de habilidades para el aprendizaje y el promedio escolar fueron significativas y positivas casi en su totalidad con una p menor de 0.05 , con un nivel de correlación entre moderada y débil, observándose que la variable Promedio escolar (PROMESC) no correlaciona con la eficiencia para escribir y con la eficiencia para pensar, por presentar en ambos casos una p mayor a .05 (ver Tabla 14).

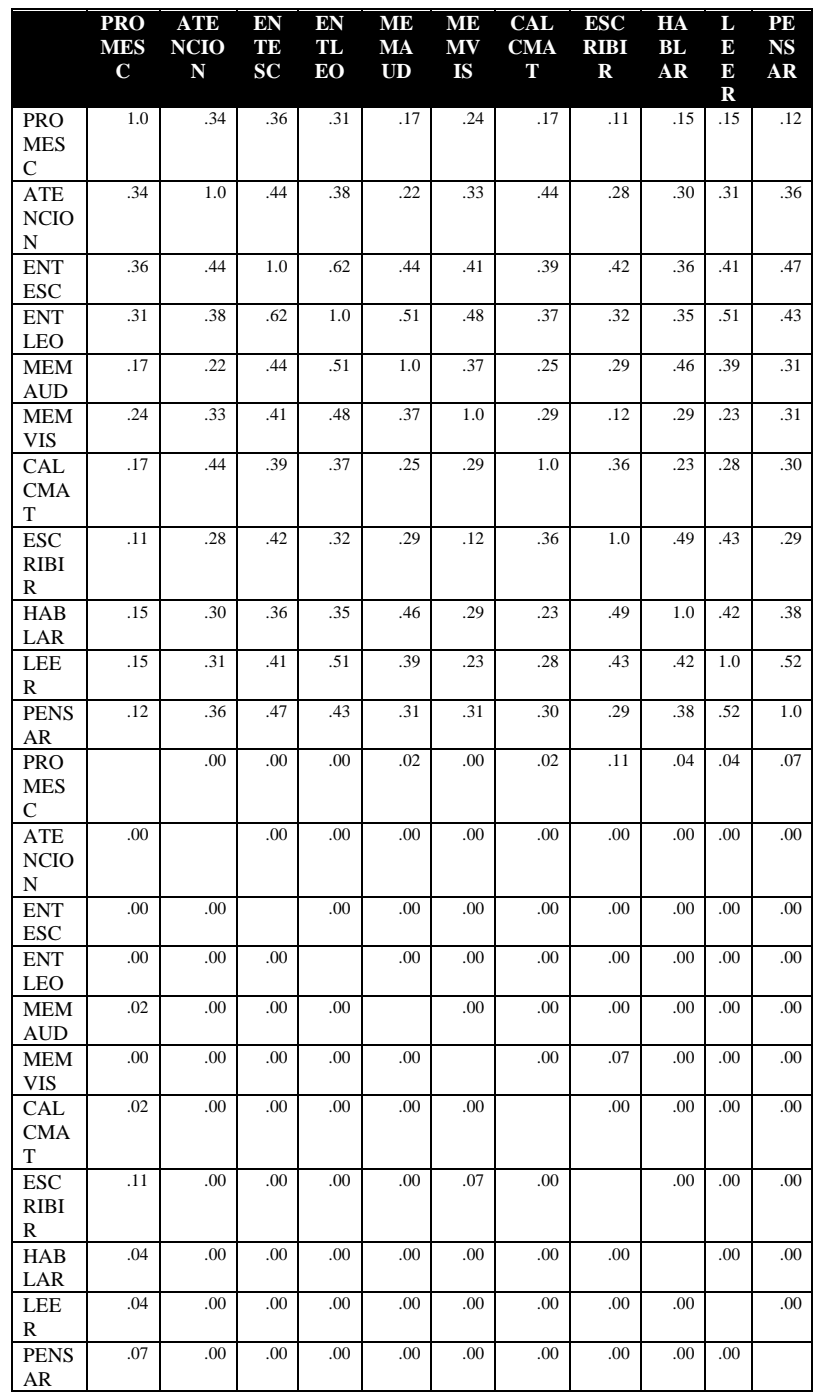

Tabla 14 Matriz de correlacionesa Autorreferencia Promedio escolar y Autoevaluación de habilidades para el aprendizaje

De la lectura de la tabla 1 , de correlaciones se puede considerar que en la medida que los estudiantes encuestados refieren mayor promedio escolar de manera débil a muy débil se refieren más eficientes para: atención $(\mathrm{r}$ $.342, \mathrm{p} .000$ ), entender lo que escuchan ( $\mathrm{r} .368$, $\mathrm{p} .000)$, entender lo que leen $(\mathrm{r} .314, \mathrm{p} .000)$, memoria auditiva ( $\mathrm{r} .176, \mathrm{p} .026)$, memoria visual ( $\mathrm{r} .242, \mathrm{p} .004)$, cálculo matemático $(\mathrm{r}$ $.174, \mathrm{p} .027)$, hablar (r .150, p .049) y leer (r $.154, \mathrm{p} .044)$. De lo anterior se destaca que la variable de habilidades del aprendizaje con mayor correlación con promedio escolar es Entender lo que escucho (r .368, p .000); la que menor correlación muestra es Hablar ( $\mathrm{r} .150, \mathrm{p}$ .049) y la variable que no correlaciona resultó que fueron dos, Escribir (r .110, p .113) y Pensar (r .129, p .077). Por último, la variable de habilidades del aprendizaje que mejor correlación muestra con las demás variables de habilidades del aprendizaje es Entender lo que escucho con Entender lo que leo (r .622, p .000). 


\section{Anexo 1:}

Instrumento de autoevaluación de habilidaes del aprendizaje en estudiantes universitarios

Facultad

o

Escuela:

\section{Estudiante}

de:

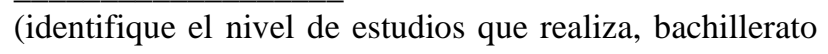
y/o que carrera o Licenciatura cursa)

IDENTIFICACIÓN: Escriba o marque el dato correspondiente

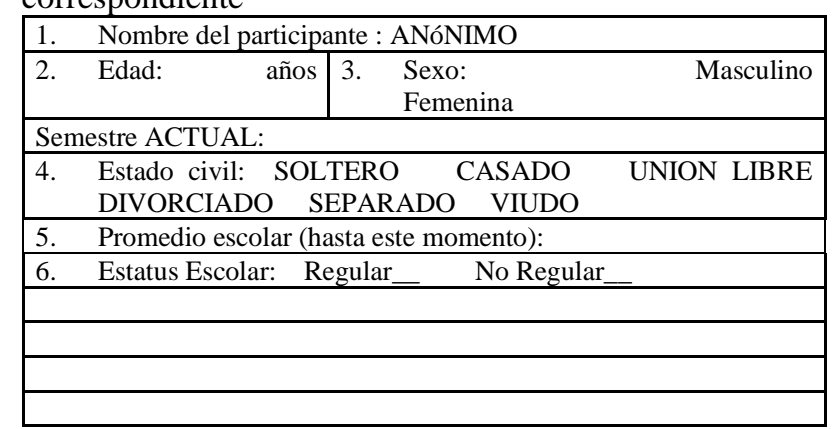

En la tabla inferior, al lado de cada enunciado, PONGA EL NÚMERO CORRESPONDIENTE a la calificación con que usted autoconsidere SU HABILIDAD usando la siguiente escala de valor:

\begin{tabular}{|l|l|l|l|l|}
\hline 0 & NADA & & \\
\hline 1 & CASI NADA & 6 & UN POCO BUENA \\
\cline { 1 - 1 } 2 & MUY DEFICIENTE & 7 & $\begin{array}{l}\text { MEDIANAMENTE } \\
\text { BUENA }\end{array}$ \\
\hline 3 & DEFICIENTE \\
$n$ & $\begin{array}{l}\text { ENTRE } \\
\text { DEFICIENTE Y } \\
\text { REGULAR }\end{array}$ & 9 & BUENA \\
\hline 5 & REGULAR & 10 & $\begin{array}{l}\text { EXCELENTEMENTE } \\
\text { BUENA }\end{array}$ \\
\hline
\end{tabular}

\begin{tabular}{|ll|l|l|l|}
\hline Habilidades: & Calificación & Habilidades: & Calificación \\
\hline 7. & Atención & $8 . \quad \begin{array}{l}\text { Cálculo } \\
\text { matemático }\end{array}$ & \\
\hline 9. & $\begin{array}{l}\text { Entender lo } \\
\text { que escucho }\end{array}$ & & 10. Escribir & \\
\hline $\begin{array}{l}\text { 11. } \\
\text { Entender lo leo }\end{array}$ & & 12. Hablar & \\
\hline $\begin{array}{l}\text { Memoria } \\
\text { AUDITIVA }\end{array}$ & & 14. Leer & \\
\hline 15. & $\begin{array}{l}\text { Memoria } \\
\text { VISUAL }\end{array}$ & 16. Pensar & \\
\hline
\end{tabular}

\section{Agradecimiento}

Se agradece a la Facultad de 'Psicología y Terapia de la Comunicación Humana, así como al Instituto de Investigación Científica de la Universidad Juárez del estado de Durango por las facilidades otorgadas para la realización de la presente investigación.

\section{Conclusión}

Los resultados obtenidos permiten definir que el instrumento de autoevaluación de las habilidades para el aprendizaje es fiable y permite identificar el nivel de eficiencia con el que se autoevalúan los participantes en la muestra estudiada, lo cual permite considerar la oportunidad de planear estrategias de atención educativa a los estudiantes universitarios a partir de su propia evaluación, quedando pendiente valorar los resultados de esta planeación y su puesta en práctica en sucesivos trabajos de investigación educativa.

\section{Referencias}

Barceló, E. L. (2006). Funciones ejecutivas en estudiantes universitarios que presentan bajo y alto rendimiento académico. Piscología desde el Caribe , 109-138.

Cronbach, L. J. (1951). Coefficient Alpha and the internal structure of tests. Psychometrika, 297-313.

Cronbach, L. J. (1972). The dependability of Behavioral Measurements: Theory of Generalizability for Scores and Profiles. New York: John Wiley \& Sons.

Cupani, M. G. (2013). El modelo de los cinco grandes factores de personalidad: contribución predictiva al desempeño académico. Revista de Psicología, 17.

Echavarri, M. G. (2007). Diferencias de género en habilidades cognitivas y rendimiento academico en estudiantes universitarios. Cordoba, Argentina.: Universidad Empresarial siglo 21 .

Gobierno de los Estados Unidos Mexicanos. Presidencia de la República. (2018). VI Informe de Gobierno 2017-2018. Cd. de México, Mexico.: Talleres Gáficos de México.

Navarro, R. E. (2003). El rendimiento académco: concepto, investigación y desarrollo. Revista Electrónica Iberoamericana sobre Calidad, Eficacia y Cambo en Educación. , sin numero de página.

Nunnally, J. (1967). Psychometric Theory. Chicago, IL.: Mc Graw Hill. 
Palacios, J. \&. (2007). Desempeño académico y conductas de riesgo en adolescentes. Revista de Educación y Desarrollo, 5-16.

Secretaria de Educacion Publica. (2014). Recuperado el 08 de Septiembre de 2017, de http://www.planeacion.sep.gob.mx/:

http://www.planeacion.sep.gob.mx/Doc/estadist ica_e_indicadores/lineamientos_formulacion_d e_indicadores.pdf

Universidad Juárez del Estado de Durango. (2017). Recuperado el 22 de 07 de 2019, de https://www.ujed.mx/:

https://www.ujed.mx/doc/publicaciones/anuario s-estadisticos/Anuario_estadistico_2016.pdf

Universidad Juárez del Estado de Durango. (2019). Recuperado el 22 de 07 de 2019, de https://www.ujed.mx/:

https://www.ujed.mx/doc/publicaciones/anuario s-estadisticos/Anuario_estadistico_2018.pdf

Universidad Juárez del Estado de Durango. (2018). https://www.ujed.mx/. Recuperado el 22 de 07 de 2019, de https://www.ujed.mx/doc/publicaciones/anuario s-estadisticos/Anuario_estadistico_2017.pdf

Velez-Van, M. R. (2005). Factors associated with academic performance in medical students. PSIC Educación Médica , 2 (8). 